explanations or details concerning the many questions which the text does not answer, one is disappointed, because they do not either. A very tiresome experience!

In his conclusion O'Connor attempts to define "good faith in international law". He states: "The principle of good faith in international law is a fundamental principle from which the rule pacta sunt servanda and other legal rules distinctively and directly related to honesty, faimess and reasonableness are derived, and the application of these rules is determined at any particular time by the compelling standards of honesty, fairness and reasonableness prevailing in the international community at the time." (p. 124) A weak statement that calls for a closer examination.

He who dares doubt that good faith indeed is a fundamental principle of international law (after all it seems to be sadly lacking in much of today's conduct of international relations) is not convinced of its existence after reading O'Connor's work.

"[...] no writer has hitherto presented a comprehensive study or proposed a definition of the principle, as it operates in international law." (p. 1) Although O'Connor proposes such a definition, a comprehensive study on good faith in international law remains a desire of legal research.

Dagmar Reimmann

\title{
Harald Hohmann
}

Präventive Rechtspflichten und -prinzipien des modernen Umweltvölkerrechts

Zum Stand des Umweltvölkerrechts zwischen Umweltnutzung und Umweltschutz Schriften zum Völkerrecht, Band 97, Duncker \& Humblot, Berlin 1992, 440 S., DM 128,--

"Umweltschutz ist eine Frage der Akzeptabilität und des Überlebens unserer Gesellschaftsordnung. Denn akzeptabel kann nur eine Gesellschaftsordnung sein, die allen Bürgern Arbeit gibt, ohne die Umwelt so zu belasten, daß sie für kommende Generationen als Lebensraum zerstört ist! Diese beiden einleitenden Aussagen (S. 19) führen Hohmann in seiner Frankfurter juristischen Dissertation (1991) ohne Umschweife zur Feststellung, dem Umweltvölkerrecht dürfe es "nicht allein um die maximale ökonomische Aufteilung natürlicher Ressourcen, sondern es muß ihm primär um optimale Ressourcenpflege gehen". Hohmann legt bei seinem Bestreben, einen allgemeinen "Perspektivwechsel von einem überwiegend ökonomischen hin zu einem primär ökologischen Ansatz" (S. 22 f.) zu belegen, drei Hauptphasen zugrunde, in die sich die historische Entwicklung des Umweltrechts einteilen lasse: Erst mit Beginn der Industrialisierung und der Massenproduktion habe eine "Verkünstlichung der Natur", eine "Natur-Ausbeutung" (S. 24) begonnen, die im nationalen (deutschen) Rahmen in den 70er Jahren von einem Ubergangszeitraum abgelöst worden sei, "mit dem Erlaß von Umwelt-Spezialgesetzen für die Umweltmedien Wasser (Boden) und 
Luft" (S. 27). Etwa ab 1986 zeige sich ein "eher medienübergreifender Ansatz mit stärkerer Betonung des Vorsorgegrundsatzes", was Hohmann als Paradigmenwechsel auffaßt: In der neuen Phase eines "Oko-Managements bzw. der planvollen Bewirtschaftung/Pflege der Natur" sei aus dem bisherigen Umweltallokations- ein Umweltschutzrecht entstanden; moderner Umweltschutz bedeute in zunehmendem Maße "vorausschauende Umweltgestaltung, Umweltplanung und nur noch zu einem geringen Teil Gefahrenabwehr" (Feldhaus, s. S. 32).

Der Frage, ob auch im Umweltvölkerrecht - die EG-Rechtslage wird nur gestreift (S. 33 N. 66) - ein paralleler Umschlag hin zu langfristiger, ökologischer Ressourcen-Pflege zu verzeichnen sei, widmet sich Hohmann auf ziemlich unkonventionelle Weise. Mehr als die Hälfte der Arbeit befaßt sich nämlich zunächst mit dem "Beitrag internationaler Organisationen und Gremien zur Entwicklung des Umweltvölkerrechts (außerhalb von Abkommen"; analysiert werden hier also Erklärungen, Resolutionen, Programme in Entwurfs- und endgültigen Fassungen, deren rechtliche Verbindlichkeit nicht gerade auf der Hand liegt, die vielmehr durchweg dem Bereich des "soft law" angehören; in einem Resümee zu diesem (2.) Kapitel erörtert Hohmann die Problematik derartiger Akte unter der befremdlichen Überschrift "methodische Vorüberlegungen" (S. 219 ff.). Unter vier "sehr engen Voraussetzungen" soll sich ein für die (völker)gewohnheitsrechtliche Geltung von Normen notwendige "(widerlegbarc) opinio iuris generalis" und damit letztlich eine Rechtspflicht für die staatlichen Völkerrechtssubjekte ergeben: Zunächst "weiche" Rechtsregeln müßten sich in einem nicht regional begrenzten Rahmen "verdichten", von einer Allgemeinheit der Staaten übernommen und in späteren Rechtsakten (nicht notwendig vertraglicher Art) wieder aufgegriffen ("zitiert") werden (S. 225 f.). Es ist daher nur konsequent, wenn Hohmann im folgenden (3.) Kapitel die diversen Abkommen zum "Schutz der Umweltmedien" aufbereitet und sie in den Raster der zuvor näher gekennzeichneten Charakteristika des modernen Umweltvölkerrechts einfügt, um auf diese Weise den Prozeß der Verfestigung von elementaren "Prinzipien bzw. Forderungen für den vorbeugenden und ressourcenschonenden Umweltschutz" (S. 246) zu veranschaulichen. In den zentralen Kapiteln 2 und 3 formuliert der Autor dabei immer wieder Zwischenergebnisse, um zu den jeweiligen Zusammenfassungen - als Basis der Schlußfolgerungen im letzten Kapitel - hinzuführen; dieses überaus eindringliche Vorgehen ruft allerdings zuweilen den Eindruck von Umständlichkeit hervor und bewirkt zwangsläufig Wiederholungen. Das seit etwa 1982/ 1987 konstatierbare (S. 410) moderne Umweltvölkerrecht weist Hohmann zufolge eine Relativität auf, die in drei Richtungen zielt: Im Hinblick auf die Rechtsquellen seien die Instrumente (förmliches) Abkommen und (informelle) Erklärung o.ä. "weitgehend austauschbar", wenngleich präzisere Formulierungen in der Regel Verträgen vorbehalten seien (S. 403); umweltrechtliche Verpflichtungen entwickelten sich immer schneller (zur Rechtsverbindlichkeit), was nicht zuletzt auf die jüngsten Kenntnisse über bevorstehende Umweltkatastrophen zurückzuführen sei (S. 404); bezüglich des gebotenen Technologiestandards spiele die Region eine wichtige Rolle, soweit keine vertraglichen Transfer- oder Finanzierungspflichten für (westliche) Industrieländer begründet seien (S. 405). Hohmann 
verkennt nicht, daß es gegenwärtig (d.h. Anfang 1991, S. 7) noch einige bedenkliche Lücken im modernen Umweltvölkerrecht gibt; die umfassende Anerkennung einer Umweltverträglichkeitsprüfung und des "sustainable use"-Ansatzes (s. S. 82 f.) ließen aber hoffen, "daß auch unsere Kinder eine Chance auf Uberleben und die Erfüllung ihrer Grundbedürfnisse haben" (S. 411). Mit diesem verhalten optimistischen Ausblick endet die Studie, der durchaus der beabsichtigte Nachweis gelungen ist, "daß das Umwelt-Gewohnheitsrecht mehr als nur Haftungsregeln kennt" (S. 9). Als nützliche Ergänzung hat Hohmann die benutzten Rechtsakte - und nicht nur sie - in einer zweibändigen Ausgabe von "Basic Documents of International Environmental Law" (1992) zusammengestellt.

Die zentrale These Hohmanns, wonach das moderne Umweltvertrags- wie -gewohnheitsrecht gekennzeichnet sei durch "unmittelbaren Schutz der Umweltmedien" (nach einem weiteren anthropozentrischen, ressourcenökonomischen Ansatz), nicht nur auf das individuelle Nachbarschaftsverhältnis sehe, sondern auf die Beziehungen zwischen mehreren, weiträumig voneinander entfernten Emissions- und Opferstaaten abstelle und schließlich in "gemeinsamen Verantwortungen", regionalen Kooperationen oder gar in globalen "common concern"-Kriterien denke (S. 378), wird nicht unangefochten bleiben, ja widerspricht wohl weithin vertretenen Auffassungen vom "Wesen" des Völkerrechts (zur Gegenposition etwa Randelzhofer, Jura 1992, S. 1 ff.). Freilich liest sich die Deklaration von Rio über Umwelt und Entwicklung vom 14.6.1992 in vielen Grundsätzen wie ein Exzerpt aus Hohmanns Werk, etwa beim Zusammenhang von Umwelt und Entwicklung oder beim Prinzip 15, wonach "der vorbeugende Ansatz von den Staaten umfassend angewandt werden" soll und dort, "wo emste und irreversible Schäden drohen, das Fehlen letzter wissenschaftlicher Sicherheit nicht dazu genutzt werden (darf), kostenintensive Maßnahmen zur Vermeidung von Umweltschäden zu verzögern". Auch die von Hohmann noch berücksichtigte (S. 345 ff.) - und in dieser Zeitschrift dargestellte (vgl. Rublack, VRÜ 22 [1989], S. 64 ff.) - Baseler Konvention über die Kontrolle des grenzüberschreitenden Transports gefährlicher Abfälle ist unlängst vom Europäischen Gerichtshof als zusätzlicher Beleg für das die EG-Umweltpolitik beherrschende Ursprungsprinzip (Art. 130 r Abs. 2 EWGV) herangezogen worden (Rs. C-2/90, DVBl. 1992, S. 1427 [1429]), wobei das Gericht für Gegenstände dieser besonderen Art - Abfälle also - eine "Gefahr für die Umwelt" bereits aus der bloßen "Ansammlung" herleite, "noch bevor sie die Gesundheit gefährden"; insoweit bestätigt die Konvention nicht allein das (lokale) "Verbot der Belastungsverschiebung" (s. Hohmann, S. 387), sondern auch die Begründung von Verhütungspflichten bei bloßem Gefahrenverdacht s. S. 381 f.). Die Neufassung des Art. 130 r EWGV durch den Maastrichter Unions-Vertrag ergänzt überdies die umweltpolitischen Ziele der Gemeinschaft um die "Förderung von Maßnahmen auf internationaler Ebene zur Bewältigung regionaler oder globaler Umweltprobleme" und führt neben Vorbeugungs-, Ursprungs- und Verursacherprinzip auch den "Grundsatz der Vorsorge" ein (Abs. 2 S. 2), eine weitere Bekräftigung des "Vorsorge-Charakters des modernen Umwelt(völker)rechts" (Hohmann, S. 406 ff.). Auch was die finanzielle Unterstützung von für bestimmte Staaten unverhältnismäßig teuren Schutzmaßnahmen anbelangt, wird der Unionsvertrag neue 
Möglichkeiten eröffnen (s. Art. 130 s Abs. 5 ebd.). Die Praxis intemationaler Organisationen also zeigt durchaus (weiterhin) Tendenzen, vermehrt auf Prävention zu setzen, auch wenn dies, wie bei der 4. UN-Konferenz zum Schutz der Ozonschicht im November 1992, halbherzig und nur langsam erfolgen mag. In einem Punkt zumindest dürfte freilich Hohmanns Sicht bis auf weiteres fragwürdig, wenn auch sympathisch bleiben - dem Postulat eines "Individualrechts gegen den eigenen Staat auf Schutz vor erheblichen Umweltbeeinträchtigungen und einem Kollektivrecht der Weltgemeinschaft auf Erhalt der international bedeutsamen Ökosysteme" (S. 388). Denn das "Recht" jedes Menschen (in Grundsatz 1 der Rio-Deklaration), "ein gesundes und produktives Leben in Einklang mit der Natur zu führen", bleibt dort konturenarm, und die "Verantwortung (der Staaten) sicherzustellen, daß Aktivitäten, die ihrer Rechtsprechung oder Kontrolle unterliegen, der Umwelt anderer Staaten oder anderer Gebiete jenseits der Grenzen nationaler Zuständigkeit keinen Schaden zufügen (Grundsatz 2 ebd.), folgt bezeichnenderweise erst nach dem Hinweis auf das "souveräne Recht" aller Staaten, "ihre eigenen Ressourcen gemäß ihrer eigenen Umwelt- und Entwicklungspolitik zu nutzen". Damit bleibt Art und Maß des Umweltschutzes primär an nationalen Interessen ausgerichtet, trotz der Verpflichtung (Grundsatz 7), "im Geiste globaler Partnerschaft zusammenzuarbeiten, um die Unversehrtheit und Integrität des Ökosystems der Erde zu bewahren, zu schützen und wiederherzustellen". Bis zu einer zentral konzipierten und durchgesetzten "Erd-Umweltpolitik" ist es wohl noch (zu ?) weit.

Ludwig Gramlich

\section{Dieter Nohlen / Franz Nuscheler (Hrsg.)}

\section{Handbuch der Dritten Welt, Band 2: Südamerika}

Verlag J.H.W. Dietz Nachf., 3. Auflage, Bonn 1992, 548 S., DM 48,--

Das von Dieter Nohlen und Franz Nuscheler 1974 erstmals herausgegebene Handbuch der Dritten Welt hat die sozialwissenschaftliche Entwicklungsforschung der letzten zwanzig Jahre in Deutschland wesentlich mitgestaltet und beeinflußt. Es ist insof ern von vornherein zu begrüßen, daß die Herausgeber zehn Jahre nach der zweiten nun eine neu bearbeitete dritte Auflage dieses Klassikers erstellt haben. Die Relevanz der Neuauflage ergibt sich nicht nur aus der (im ersten von insgesamt acht Bänden reflektierten) Fortentwicklung der theoretischen Diskussion, sondern auch und besonders aus den konkreten politischen und wirtschaftlichen Veränderungen in der sog. "Dritten Welt". Eine besondere Dynamik weisen in dieser Hinsicht die Länder Südamerikas auf, die im zweiten, hier besprochenen Band des Handbuches analysiert werden (Mittelamerika und die Karibik werden im dritten Band behandelt): Die 80er Jahre waren für die Region politisch wie auch ökonomisch ein ausgesprochen ereignisreiches Jahrzehnt. 